\title{
Fabrication of Submicron Thermosetting Carbon Nanotube-Epoxy Fibers Using Electrospinning
}

\author{
NOJAN ALIAHMAD ${ }^{a}$, VIDYA WABLE ${ }^{a, b}$, \\ PIAS KUMAR BISWAS ${ }^{a, b}$, IRAN HERNANDEZ ${ }^{a}$, \\ HAMID DALIR ${ }^{\mathrm{a}, \mathrm{b},{ }^{*}}$ and MANGILAL AGARWAL ${ }^{\mathrm{a}, \mathrm{b},{ }^{*}}$
}

\begin{abstract}
Recently epoxy-based nanocomposites are gaining tremendous attention in many structural applications such as those in aerospace, automotive and motorsports. This research represents a new approach to fabricate submicron thermoset epoxy filaments enhanced with carbon nanotubes (CNT), through optimized curing followed by an electrospinning process. The optimized curing process is based on the uniform mixing of CNT with epoxy, and partial curing of the CNT/epoxy mixture with the hardener through a thermal treatment without adding any plasticizers or thermoplastic binders. Later the fibers have been made by electrospinning of the semi-cured mixture. Fig 1 shows the fabrication process of the described filaments. The key goal is to make the thermosetting epoxy without adding any thermoplastic to keep the integrity and quality of the fibers. The diameters of these filaments can be tuned between 100 $\mathrm{nm}$ to $500 \mathrm{~nm}$. Further, the CNT structure has been aligned inside the filament structure by the presence of the electrostatic field during the electrospinning process results in better stability and smaller diameters for the fibers. The fabricated filaments show that adding a low amount of CNT in the epoxy structure, better structural, electrical and thermal stability, has been achieved.
\end{abstract}

\section{INTRODUCTION}

By improvements on nanofabrication and development of nanomaterials, advancements have been achieved on nanocomposite. Development of submicron filaments for composite applications is one of the desired areas of investigation to achieve higher strengths and mechanical stability. In this approach submicron filaments are extruded form a polymer achieving high surface area and uniformity. The developed filaments can also be reinforced with nanomaterials, to be used as

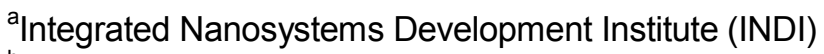

${ }^{b}$ Purdue School of Engineering and Technology

*Indiana University-Purdue University, Indianapolis, IN 46202, USA
} 
reinforcements, sensing materials and shielding layers [1, 2]. One of the desired methods to fabricate these submicron filaments is electeospinning. Compared to other continues extrusion approaches to make submicron filaments, electrospinning, is widely acknowledged as the most versatile, effective and economically beneficial process. This is a simple method based on the electrohydrodynamic phenomena, which is a voltage-driven approach to extrude filament [3]. The simplicity of fabrication is the main benefit of applying this technique. Thus, Voltage, flow rate, needle-collector distance, and viscosity, are all represent key parameters in regulating the properties of the fibers created through electrospinning [4, 5]. The uniform polymer nanofibers structures formed by electrospinning have been reported to have diameters ranging couple of nanometers to several microns and exhibit superior properties like the high surface area to volume ratio, flexibility in surface functionalities, inter/intra fibrous porosity, and extraordinary mechanical properties [6].

Further, adding nanomaterials to the composite enhances the mechanical and electrical properties of them. Thus, making the fibers using nanocomposite leads to achieve high strength filaments with improved properties. CNT is one of the best nanomaterials to be used for composite applications, while it has tremendous mechanical and electrical properties [7, 8]. Nevertheless, there are still preventive aspects that limits applying CNT in polymer nanocomposites. These factors are lack of solubility, complex fabrication process and phase separation [9]. Due to these limiting factors having a CNT-based composite is still a challenge.

The goal of this work is to fabricate submicron fibers using electrospinning along with well mixed and uniform CNT nano reinforcements. To also make these fibers compatible with Carbon Fiber Reinforced Polymer (CFRP) composites, thermosetting epoxy has been used as the base. The fabrication of a filament using a thermosetting polymer is still a challenge due to the nature of this epoxy $[10,11]$. While the base of electospinning is the extrusion of a highly viscous polymer, most of the thermosetting polymers (epoxy) are limited to be extruded or stretched. Thus, fabrication of a uniform micro or nanoscale filament out of epoxy is still a challenge. Further, due to high viscosity of thermosetting polymers, adding any nanomaterials such as carbon nanotubes can generate clusters in the structure, reducing the uniformity and making it less suitable for electrospinning [12].

In this manuscript a novel approach has been developed based on elctrospinning to overcome the fabrication limits of thermosetting epoxy submicron filaments. Thus, the main goal is to create fibers that could be electrospun along with nano reinforcements (CNT) to be used with epoxy matrices in advanced diversified composite applications.

\section{METHODS}

The CNT/epoxy solution has been made by Bisphenol A (50 - 99 pbw. \%) epoxy and multi walled carbon nanotubes (5 wt. \%). Dimethylformamide (DMF) (1:4 volume ratio) and Triton X- 100 (20:1 volume ratio) were added to the mixture, as solvent and surfactant accordingly. The mixture was stirred using a magnet stirrer for 4 hours and then sonicated for 10 mins to achieve a well-mixed and uniform solution. 
After that to adjust the CNT percentage, more epoxy was added to the masterbatch and mixed for another 15 mins, followed by sonication. In the last step to have a uniform viscous mixture for electrospinning, the curing agent was mixed at a ratio of 15:1 and stirred at $50^{\circ} \mathrm{C}$ for 2 hours to make a partially cured solution. This solution was rested fort 16 hours to become stable for electrospinning.

For electrospinning, the CNT/epoxy mixture was added to a syringe with a needle gauge of $26 \mathrm{G}$. The pumping rate of the epoxy solution was adjusted to $0.5 \mathrm{~mL} / \mathrm{hr}$. The electrospinning voltages of $12-16 \mathrm{kV}$ was applied between the needle and collector at room temperature and a needle tip/collector distance of $10 \mathrm{~cm}$. Figure 1 shows the fabrication process of the submicron filaments.

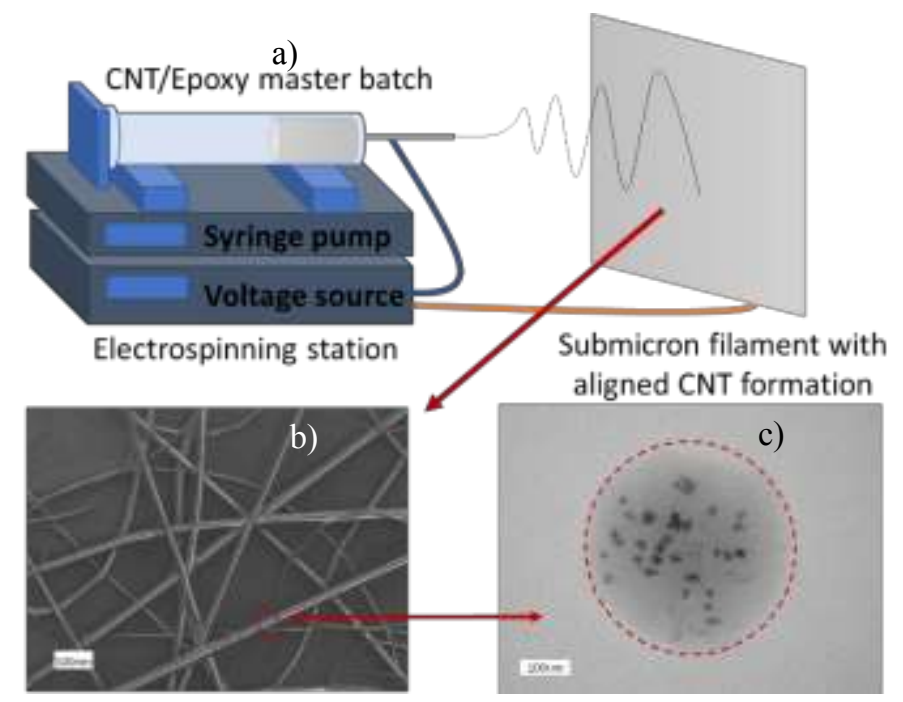

Figure 1. a) Process schematic for making submicron CNT/Epoxy filament through electrospinning, b) SEM and c) TEM images of the fabricated fibers.

\section{RESULTS AND DISCUSSION}

The CNT/epoxy batch was observed after preparation and showed no phase separation after 24 hours, and the solution was still dark black after adding the CNT. To achieve a spinnable viscosity, the epoxy mixture was adjusted using a partial curing method. The partial curing of the thermosetting epoxy helped to achieve spinnable viscosity to make it spinnable, but not completely cured. To check the process, samples were made with different resting times $(5,20$, and $30 \mathrm{hrs})$ and the quality of the resulting fibers was investigated. It was found that the lowest resting time (5hrs) was not enough to ensure proper chemical bonding between the epoxy and hardener, thus the viscosity is still in the range of $5 \mathrm{p}$. The higher rest time $(30 \mathrm{hrs}$ and more) almost fully cures the epoxy and results in high viscosities in the range of $500 \mathrm{p}$, preventing the solution to be spinnable. By curing the epoxy solution for too long, higher ratios of entangles affect the uniformity of the fibers and lead to the production of large bids. In this case the 20 hours of resting time is the optimum while the viscosity of the solution reached to $65 \mathrm{p}$ makes it suitable for electrospinning. Figure 1a shows the electospunned submicron filament after resting 20 hours. 
The uniformity of the electrospun nanofibers was observed using field emission scanning electron microscopy (FESEM) and transmission electron microscopy (TEM). Figure 2a shows a cross-section FESEM image of a spunned filament and Figure $2 b$ shows the top view of the deposited filament layer. As it is shown here the thickness of the fibers are in the range of 100-500 nm and a uniform one directional CNT network has been formed inside the fibers. Figure $2 \mathrm{c}$ also reveals the TEM crosssection of a CNT/epoxy fiber. As it was observed CNT rods are forming a unidirectional formation inside the epoxy structure. The formation of aligned CNT network inside the epoxy structure is due to the uniformity of the CNT/epoxy mixture followed by electrospinning. The presence of electric field in this process aligns the highly conductive CNT nanorods. Further while the length of the CNT is in the range of 2 to $3 \mu \mathrm{m}$ (which is 20 times larger than the width of the filaments) the CNT rods have to align in the direction of the CNT/epoxy filament.
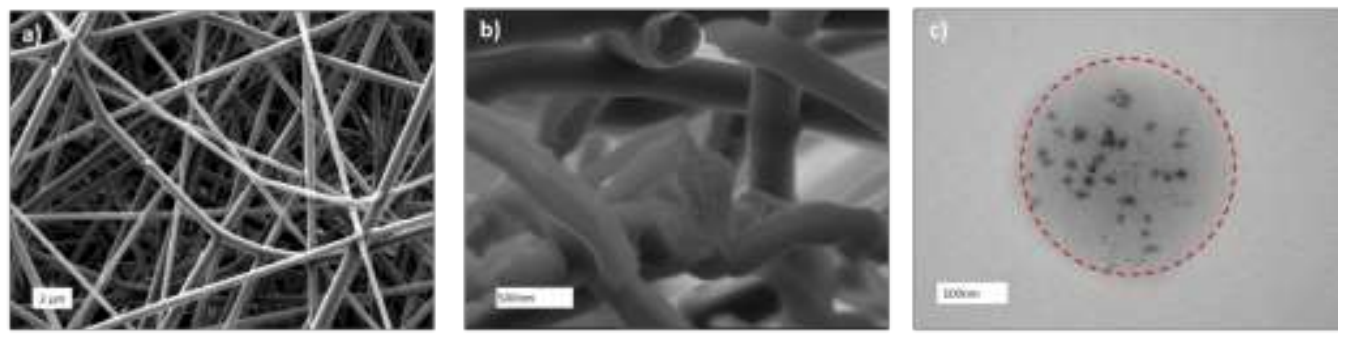

Figure 2. FESEM image of a) surface and b) cross-section of the submicron CNT/epoxy fiber, c) side view TEM image of the of the electrospun CNT/epoxy fiber revealing the formation of aligned CNT network inside the structure.

Mechanical properties of the filaments also were tested using atomic force microscopy. Epoxy-based submicron filaments with $0 \%, 2 \%$ and $4 \%$ CNT have been made though electrospinnig and the modulus of the filaments were tested. As it was observed (Figure 3a) the mechanical properties have been improved by adding more CNT to the structure. while adding more than $4 \%$ CNT makes clusters and reduce the uniformity of the submicron filaments, the maximum CNT percentage was limited to $4 \%$ in this section. The modulus has been increased from $3.24 \mathrm{GPa}$ for epoxy filaments to $4.2 \mathrm{GPa}$ and $4.84 \mathrm{GPa}$ for $2 \%$ and $4 \% \mathrm{CNT}$ samples respectively. The thermal properties of the fibers also have not been changed by adding more CNT. It was realized that by adding CNT the crystallinity of the epoxy has been improved and it has little effect on the decomposition temperature of the epoxy and does not provide any thermal instability. Here by adding CNT the degradation temperature starts earlier, while complete decomposition of is in higher temperature, compared to the samples with near epoxy. 


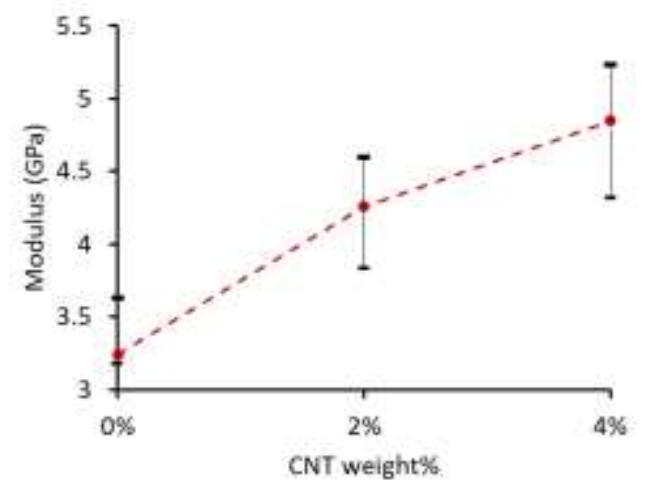

Figure 3. Modulus Volume fraction relation of the CNT/epoxy nanofiber with different CNT concentrations.

\section{CONCLUSION}

This research represents a novel method to develop submicron epoxy-based filaments enhanced with CNT. This is bases on optimized curing process of CNT/epoxy and homogenous mixing of CNT in epoxy medium. The partial curing of epoxy with thermal treatment process makes the composite viscous enough, suitable for electrospinning. Using this method, the fibers have been made without adding any plasticizers or thermoplastic binders through electrospinning process. The diameters of these filaments can be adjusted between $100 \mathrm{~nm}$ to $500 \mathrm{~nm}$. Further, adding the CNT also improved mechanical and thermal properties of the filaments due to the formation of aligned CNT network inside the filament structure. The study shows that the fibers have better crystalline structure and improved modulus (up to 59\%) by adding only $4 \%$ CNT. Adding a very limited amount of CNT in the epoxy structure makes this submicron filaments suitable for many applications such as aerospace reinforcement.

\section{ACKNOWLEDGMENTS}

The authors would like to express their gratitude to the National Science Foundation Major Research Instrumentation Program by supporting this research (Award \# 1229514) for the FESEM. Any opinions, findings, and conclusions or recommendations expressed in this material are those of the author(s) and do not necessarily reflect the views of the National Science Foundation. The authors would also like to thank Dr. Daniel Minner, for his assistance with the instrumentation and proofreading, Reza Moheimani and Nahal Aliheidari for lab assistance.

\section{REFERENCES}

1. Wang, Y., et al., Ultrastable, highly luminescent organic-inorganic perovskite-polymer composite films. Advanced Materials, 2016. 28(48): p. 10710-10717.

2. Ling, T., et al., Freestanding ultrathin metallic nanosheets: Materials, synthesis, and applications. Advanced Materials, 2015. 27(36): p. 5396-5402. 
3. Demir, M.M., et al., Mechanical interlocking between porous electrospun polystyrene fibers and an epoxy matrix. ACS applied materials \& interfaces, 2014. 6(24): p. 2190121905.

4. Reneker, D.H. and A.L. Yarin, Electrospinning jets and polymer nanofibers. Polymer, 2008. 49(10): p. 2387-2425.

5. Theron, S., E. Zussman, and A. Yarin, Experimental investigation of the governing parameters in the electrospinning of polymer solutions. Polymer, 2004. 45(6): p. 20172030.

6. Aliheidari, N., et al., Electrospun Nanofibers for Label-Free Sensor Applications. Sensors, 2019. 19(16): p. 3587.

7. Fiedler, B., et al., Fundamental aspects of nano-reinforced composites. Composites science and technology, 2006. 66(16): p. 3115-3125.

8. Xie, X.-L., Y.-W. Mai, and X.-P. Zhou, Dispersion and alignment of carbon nanotubes in polymer matrix: a review. Materials science and engineering: R: Reports, 2005. 49(4): p. 89-112.

9. $\quad$ Song, Y.S. and J.R. Youn, Influence of dispersion states of carbon nanotubes on physical properties of epoxy nanocomposites. Carbon, 2005. 43(7): p. 1378-1385.

10. Rosso, P., et al., A toughened epoxy resin by silica nanoparticle reinforcement. Journal of Applied Polymer Science, 2006. 100(3): p. 1849-1855.

11. Johnsen, B., et al., Toughening mechanisms of nanoparticle-modified epoxy polymers. Polymer, 2007. 48(2): p. 530-541.

12. Cheng, Q., et al., Carbon nanotube/epoxy composites fabricated by resin transfer molding. Carbon, 2010. 48(1): p. 260-266. 